\title{
VANISHING VISCOSITY LIMIT TO RAREFACTION WAVES FOR THE NAVIER-STOKES EQUATIONS OF ONE-DIMENSIONAL COMPRESSIBLE HEAT-CONDUCTING FLUIDS*
}

\author{
SONG JIANG ${ }^{\dagger}$, GUOXI NI ${ }^{\ddagger}$, AND WENJUN SUN $§$
}

\begin{abstract}
We prove the solution of the Navier-Stokes equations for one-dimensional compressible heat-conducting fluids with centered rarefaction data of small strength exists globally in time, and moreover, as the viscosity and heat-conductivity coefficients tend to zero, the global solution converges to the centered rarefaction wave solution of the corresponding Euler equations uniformly away from the initial discontinuity.
\end{abstract}

Key words. Compressible Navier-Stokes equations, vanishing viscosity limit, rarefaction waves, Euler equations

AMS subject classifications. 76N17, 35M10, 35Q30, 35L65, 35L45, 35B40

1. Introduction and the main result. We study the asymptotic behavior, as the viscosity and heat-conductivity go to zero, of solutions to the Cauchy problem for the Navier-Stokes equations for a one-dimensional compressible heat-conducting fluid (in Lagrangian coordinates):

$$
\left\{\begin{array}{l}
v_{t}-u_{x}=0 \\
u_{t}+p_{x}=\epsilon\left(\frac{u_{x}}{v}\right)_{x} \\
\left(e+\frac{u^{2}}{2}\right)_{t}+(u p)_{x}=\left(\kappa \frac{\theta_{x}}{v}+\epsilon \frac{u u_{x}}{v}\right)_{x}
\end{array}\right.
$$

with (discontinuous) initial data

$$
(u, v, e)(x, 0)=\left(u_{0}, v_{0}, e_{0}\right)(x), \quad x \in \mathbb{R},
$$

where $v, u, \theta, p=p(e, v)$ and $e$ denote the specific volume, the velocity, the temperature, the pressure and the internal energy respectively, and $\epsilon, \kappa$ are the viscosity and heat conductivity coefficients, respectively. At infinity, the initial data $u_{0}, v_{0}, e_{0}$ are assumed to satisfy

$$
\lim _{x \rightarrow \pm \infty}\left(u_{0}, v_{0}, e_{0}\right)(x)=\left(u_{ \pm}, v_{ \pm}, e_{ \pm}\right)
$$

where $u_{ \pm}, v_{ \pm}$and $e_{ \pm}$are given constant states.

The system (1.1), describing the motion of the fluid, are the conservation laws of mass, momentum and energy.

The asymptotic behavior of viscous flows, as the viscosity vanishes, is one of the important topics in the theory of compressible flows. It is expected that a general weak entropy solution to the Euler equations should be (strong) limit of solutions to

\footnotetext{
*This work was supported by the Special Funds for Major State Basic Research Projects, the NSFC (Grant No. 10225105) and the CAEP (Grant No. 2003-R-02) of China.

${ }^{\dagger}$ LCP, Institute of Applied Physics and Computational Mathematics (jiang@iapcm.ac.cn).

${ }^{\ddagger}$ Corresponding author, LCP, Institute of Applied Physics and Computational Mathematics (gxni@iapcm.ac.cn).

${ }^{\S}$ LCP, Institute of Applied Physics and Computational Mathematics (sun_wenjun@iapcm.ac.cn)
} 
the corresponding Navier-Stokes equations with same initial data as the viscosity and heat conductivity tend to zero.

For the one-dimensional compressible isentropic Navier-Stokes equations

$$
\left\{\begin{array}{l}
v_{t}-u_{x}=0, \\
u_{t}+p(v)_{x}=\epsilon\left(\frac{u_{x}}{v}\right)_{x},
\end{array}\right.
$$

and the corresponding inviscid $p$-system

$$
\left\{\begin{array}{l}
v_{t}-u_{x}=0 \\
u_{t}+p(v)_{x}=0
\end{array}\right.
$$

the vanishing viscosity limit for the Cauchy problem has been studied by several researchers. In [7] DiPerna uses the method of compensated compactness and established a.e. convergence of admissible solutions $\left(u^{\epsilon}, v^{\epsilon}\right)$ of (1.4) to an admissible solution of (1.5), provided that $\left(u^{\epsilon}, v^{\epsilon}\right)$ is uniformly $L^{\infty}$ bounded and $v^{\epsilon}$ is uniform bounded away from zero. However, this uniform boundedness is difficult to verify in general, and the abstract analysis in [7] gets little information on the qualitative nature of the viscous solutions. In [14] Hoff and Liu investigate the inviscid limit problem for (1.4) in the case that the underlying invscid flow is a single weak shock wave, and they show that solutions of the compressible Navier-Stokes equations with shock data exist and converge to the inviscid shocks, as viscosity vanishes, uniformly away from the shocks. Based on [9, 14], Xin in [30] shows that the solution to the Cauchy problem for the system (1.4) with weak centered rarefaction wave data exists for all time and converges to the weak centered rarefaction wave solution of the corresponding Euler equations, as the viscosity tends to zero, uniformly away from the initial discontinuity. Moreover, for a given centered rarefaction wave to the Euler equations with finite strength, he constructs a viscous solution to the compressible Navier-Stokes system with initial data depending on the viscosity, such that the viscous solution approaches the centered rarefaction wave as the viscosity goes to zero at the rate $|\ln \epsilon| \epsilon^{1 / 4}$ uniformly for all time away from $t=0$. In the vanishing viscosity limit, the Prandtl boundary layers (characteristic boundaries) are studied for the multidimensional linearized compressible Navier-Stokes equations by using asymptotic analysis in $[31,32,29]$, while the boundary layer stability in the case of non-characteristic boundaries and one spatial dimension is discussed in [26, 23]. We mention that there is an extensive literature on the vanishing artificial viscosity limit for hyperbolic systems of conservation laws, see, for example, $[7,8,9,18,17,33,10,25,3,11,12,1]$, also cf. the monographs $[2,5,24]$ and the references therein. We also mention that the convergence of 1-d Broadwell model and the relaxation limit of a rate-type viscoelastic system to the isentropic Euler equations with centered rarefaction wave initial data are studied in $[28,15]$, respectively.

Our aim in this paper is to study the relation between the solution $\left(u^{\epsilon}, v^{\epsilon}, e^{\epsilon}\right)(x, t)$ of the Navier-Stokes equations for a compressible heat-conducting fluid (1.1) and the solution $(u, v, e)(x, t)$ of the corresponding inviscid Euler equations:

$$
\left\{\begin{array}{l}
v_{t}-u_{x}=0 \\
u_{t}+p_{x}=0 \\
\left(e+\frac{u^{2}}{2}\right)_{t}+(u p)_{x}=0
\end{array}\right.
$$


with the initial data

$$
(u, v, e)(x, 0)=\left(\tilde{u}_{0}, \tilde{v}_{0}, \tilde{e}_{0}\right)(x), \quad x \in \mathbb{R},
$$

satisfying

$$
\lim _{x \rightarrow \pm \infty}\left(\tilde{u}_{0}, \tilde{v}_{0}, \tilde{e}_{0}\right)(x)=\left(u_{ \pm}, v_{ \pm}, e_{ \pm}\right)
$$

with the same constant states $\left(u_{ \pm}, v_{ \pm}, e_{ \pm}\right)$as in (1.3).

It is convenient to work with the equations for the entropy $s$ and the absolute temperature $\theta$. The second law of thermodynamics asserts that

$$
\theta d s=d e+p d v
$$

We assume, as is customary in thermodynamics, that given any two of thermodynamics variables $\rho, e, \theta, s$ and $p$, we can obtain the remaining three variables. If we choose $(v, \theta)$ as independent variables and write $(p, e, s)=(p, e, s)(v, \theta)$, we deduce that

$$
s_{v}(v, \theta)=p_{\theta}(v, \theta), \quad s_{\theta}(v, \theta)=\frac{e_{\theta}(v, \theta)}{\theta}, e_{v}(v, \theta)=\theta p_{\theta}(v, \theta)-p(v, \theta) .
$$

Then, a straightforward calculation gives

$$
\begin{aligned}
& s_{t}=\kappa\left(\frac{\theta_{x}}{v \theta}\right)_{x}+\kappa \frac{\theta_{x}^{2}}{v \theta^{2}}+\epsilon \frac{u_{x}^{2}}{v \theta}, \\
& \theta_{t}+\frac{\theta p_{\theta}(v, \theta)}{e_{\theta}(v, \theta)} u_{x}=\frac{\kappa}{e_{\theta}(v, \theta)}\left(\frac{\theta_{x}}{v}\right)_{x}+\frac{\epsilon}{e_{\theta}(v, \theta)} \frac{u_{x}^{2}}{v} .
\end{aligned}
$$

We may also choose $(v, s)$ as independent variables and write

$$
p=p(v, s), \quad \theta=\theta(v, s) .
$$

Thus, instead of (1.1), we shall study the system $(1.1)_{1},(1.1)_{2}$ and $(1.9)$, or $(1.1)_{1},(1.1)_{2}$ and (1.10). Namely, we shall consider

$$
\left\{\begin{array}{l}
v_{t}-u_{x}=0 \\
u_{t}+p(v, s)_{x}=\epsilon\left(\frac{u_{x}}{v}\right)_{x}, \\
s_{t}=\kappa\left(\frac{\theta_{x}}{v \theta}\right)_{x}+\kappa \frac{\theta_{x}^{2}}{v \theta^{2}}+\epsilon \frac{u_{x}^{2}}{v \theta},
\end{array}\right.
$$

with initial data

$$
(u, v, s)(x, 0)=\left(u_{0}, v_{0}, s_{0}\right)(x)= \begin{cases}\left(u_{-}, v_{-}, s_{-}\right), & x<0 \\ \left(u_{+}, v_{+}, s_{+}\right), & x>0\end{cases}
$$

where $u_{ \pm}, v_{ \pm}$and $s_{ \pm}$are the constant states. The corresponding inviscid Euler equations read:

$$
\left\{\begin{array}{l}
v_{t}-u_{x}=0 \\
u_{t}+p(v, s)_{x}=0 \\
s_{t}=0
\end{array}\right.
$$


We assume in this paper that the pressure $p$ is a smooth function of its arguments satisfying

$$
p_{v}(v, s)<0<p_{v v}(v, s) \quad \text { for } \quad v>0 .
$$

Notice that the condition (1.14) assures the system (1.13) has characteristic speeds

$$
\lambda_{1}=-\sqrt{-p_{v}}, \quad \lambda_{2}=0, \quad \lambda_{3}=\sqrt{-p_{v}},
$$

and there are two family of rarefaction waves for the Euler equations (1.13). For illustration, we describe only the 1-rarefaction waves, and thus assume $s^{+}=s_{-} \equiv \bar{s}$. The case for the 3 -rarefaction waves can be dealt with similarly.

Suppose the end states $\left(u_{ \pm}, v_{ \pm}, \bar{s}\right)$ can be connected by 1-rarefaction waves. The centered 1-rarefaction wave connecting $\left(u_{-}, v_{-}, \bar{s}\right)$ to $\left(u_{+}, v_{+}, \bar{s}\right)$ is the self-similar solution $(u, v, s)(x, t)=\left(u^{r}, v^{r}, s^{r}\right)(x / t)$ of (1.13) defined by (see, e.g., [27, 4])

$$
\left\{\begin{array}{l}
s^{r}(\xi)=\bar{s} \\
u^{r}(\xi)=u_{-}+\int_{v_{-}}^{v^{r}(\xi)} \lambda_{1}(z, \bar{s}) d z \\
\lambda_{1}\left(v^{r}, \bar{s}\right)(x, t) \text { increasing in } x, \quad \lambda_{1}\left(v^{r}, \bar{s}\right)(x, t)=-\sqrt{-p_{v}\left(v^{r}(x / t), \bar{s}\right)}
\end{array}\right.
$$

which is uniquely determined by the system (1.13) and the rarefaction wave initial data

$$
\left.(u, v, s)\right|_{t=0} \equiv\left(u_{0}^{r}, v_{0}^{r}, s_{0}^{r}\right)(x)= \begin{cases}\left(u_{-}, v_{-}, \bar{s}\right), & x<0 \\ \left(u_{+}, v_{+}, \bar{s}\right), & x>0 .\end{cases}
$$

For the internal energy $e(v, \theta)$, the viscosity and heat-conductivity coefficients $\epsilon, \kappa$, we assume that for some constant $C>0$,

$$
\left\{\begin{array}{l}
e_{\theta}(v, \theta)>0 \quad \text { for } v, \theta>0, \\
\kappa=O(\epsilon) \text { as } \epsilon \rightarrow 0, \quad \kappa(\epsilon) / \epsilon \geq C>0 \text { for all } \epsilon>0 .
\end{array}\right.
$$

From the kinetic theory, the viscosity and heat-conductivity should be in the same order. In this sense, the assumption $\kappa=O(\epsilon)$ in (1.17) is reasonable.

For the sake of convenience, throughout this paper we denote

$$
\alpha=\left|u_{+}-u_{-}\right|+\left|v_{+}-v_{-}\right| .
$$

In this paper, we prove that the solution of system (1.11) with the centered rarefaction wave initial data (1.16) of small strength $\alpha$ exists for all time and converges to the centered rarefaction wave of the Euler equation (1.13) as $\epsilon \rightarrow 0$ uniformly away from the initial discontinuity. More precisely, the main result of this paper reads:

THEOREM 1.1. Let the constant states $\left(u_{ \pm}, v_{ \pm}, \bar{s}\right)$ be connected by a centered 1-rarefaction wave $\left(u^{r}\left(\frac{x}{t}\right), v^{r}\left(\frac{x}{t}\right), s^{r}\left(\frac{x}{t}\right)\right)$ defined by (1.15). Assume that (1.14) and (1.17) hold. Then, for $\alpha$ small enough, the compressible Navier-Stokes equations (1.11) with the rarefaction wave initial data (1.16) have a global piecewise smooth solution $\left(u^{\epsilon}(x, t), v^{\epsilon}(x, t), s^{\epsilon}(x, t)\right)$, such that

(i) $u^{\epsilon}, \theta^{\epsilon}$ are continuous for $t>0, v^{\epsilon}$ and $u_{x}^{\epsilon}, v_{x}^{\epsilon}, \theta_{x}^{\epsilon}$ are uniformly Hölder continuous in the set $x<0, t \geq \tau$ and $x>0, t \geq \tau$ for any $\tau>0 ; u_{t}^{\epsilon}, u_{x x}^{\epsilon}, v_{x t}^{\epsilon}, \theta_{t}^{\epsilon}, \theta_{x x}^{\epsilon}$ are 
Hölder continuous on compact set $(x, t), x \neq 0, t>0$. Moreover, the jumps in $v^{\epsilon}$ at $x=0$ satisfy

$$
\left|\left[v^{\epsilon}(0, t)\right]\right| \leq C_{1} \exp \left(-C_{2} t / \epsilon\right)
$$

and so does the other jumps, where $C_{1}, C_{2}$ are positive constants independent of $t$ and $\epsilon$, and $[\cdot]$ denotes jumps in what follows.

(ii) The solution $\left(u^{\epsilon}, v^{\epsilon}, s^{\epsilon}\right)$ converges to the centered rarefaction wave $\left(u^{r}, v^{r}, s^{r}\right)$ as $\epsilon \rightarrow 0$ uniformly away from $t=0$, i.e., for any positive $h$, we have

$$
\lim _{\epsilon \rightarrow 0} \sup _{x \in \mathbb{R}, t>h}\left|\left(u^{\epsilon}(x, t), v^{\epsilon}(x, t), s^{\epsilon}(x, t)\right)-\left(u^{r}\left(\frac{x}{t}\right), v^{r}\left(\frac{x}{t}\right), s^{r}\left(\frac{x}{t}\right)\right)\right|=0 .
$$

(iii) For any fixed viscosity $\epsilon>0$, the solution $\left(u^{\epsilon}, v^{\epsilon}, s^{\epsilon}\right)$ approaches the centered rarefaction wave $\left(u^{r}, v^{r}, s^{r}\right)$ uniformly as time goes to infinity, i.e.,

$$
\lim _{t \rightarrow \infty} \sup _{x \in \mathbb{R}^{1}}\left|\left(u^{\epsilon}(x, t), v^{\epsilon}(x, t), s^{\epsilon}(x, t)\right)-\left(u^{r}\left(\frac{x}{t}\right), v^{r}\left(\frac{x}{t}\right), s^{r}\left(\frac{x}{t}\right)\right)\right|=0 .
$$

REMARK 1.1. i) The exponential decay with respect to $t$ of the jumps in $v^{\epsilon}$ also remains valid for $\left[u_{x}^{\epsilon}\right]$ and $\left[\theta_{x}^{\epsilon}\right]$.

ii) The smallness of $\alpha$ is needed in (2.11) in Section 2 to make $\left\|\varphi_{0 y}\right\|_{ \pm}$small (cf. Remark 2.1).

To prove Theorem 1.1 and to overcome the difficulties induced by non-isentropy of the flow, we shall adapt and modify the arguments in $[30,13,22]$. Namely, we first use a natural scaling argument to reduce the proof to the nonlinear time-asymptotic stability analysis of rarefaction waves for the compressible Navier-Stokes equation (1.11) under non-smooth initial perturbations. Then, observing that the approximation of the smooth rarefaction waves to the rarefaction wave of Euler equation depends on both the strength and the initial perturbation, we exploit the smoothing property induced by the parabolic parts in (1.12) and the smallness of $\alpha$, and employ delicate energy estimates and control carefully jumps to obtain the theorem.

We point out here that in view of Theorem 1.1, an initial jump discontinuity at $x=0$ can be allowed in (1.2). The evolution of this jump discontinuity is an important aspect in our analysis. It has been shown in [13] that the discontinuity evolution follows a curve $\dot{x}=-[u] /[v]$ in $x$-t plane, and the jump discontinuity in $v, u_{x}$ and $\theta_{x}$ decays exponentially in time, while the discontinuity in $u$ and $\theta$ are smoothed out at positive time, see [13] for details. We shall exploit this fact in the proof of Theorem 1.1.

In Section 2 we reformulate the problem and give the proof of Theorem 1.1, while Section 3 is dedicated to the derivation of a priori estimates used in Section 2.

Throughout this paper, we use the following notation:

$$
\begin{aligned}
& \mathbb{R}^{-}:=(-\infty, 0), \quad \mathbb{R}^{+}:=(0, \infty), \quad\|\cdot\| \equiv\|\cdot\|_{L^{2}(\mathbb{R})}, \quad\|\cdot\|_{L^{p}} \equiv\|\cdot\|_{L^{p}(\mathbb{R})}, \\
& \|\cdot\|_{ \pm}^{2} \equiv\|\cdot\|_{L^{2}\left(\mathbb{R}^{-}\right)}^{2}+\|\cdot\|_{L^{2}\left(\mathbb{R}^{+}\right)}^{2}, \quad \int_{ \pm} \cdot d y \equiv \int_{-\infty}^{0} \cdot d y+\int_{0}^{\infty} \cdot d y .
\end{aligned}
$$

2. Reformulation and the proof of Theorem 1.1. In this section, we will reduce the proof of Theorem 1.1 to the nonlinear time-asymptotic stability analysis of rarefaction waves for the system (1.11) under non-smooth perturbations. 
First, we derive some necessary estimates on the rarefaction waves of the Euler equations (1.13) based on the inviscid Burgers equation, in particularly, we construct an explicit smooth 1-rarefaction wave which well approximates a given centered 1rarefaction wave. We start with the Riemann problem for the Burgers equation:

$$
\left\{\begin{array}{l}
w_{t}+\left(\frac{w^{2}}{2}\right)_{x}=0 \\
w(x, 0)=w_{0}^{r}(x)
\end{array}\right.
$$

where $w_{0}^{r}(x)$ is given by

$$
w_{0}^{r}(x)= \begin{cases}w_{-}, & x<0, \\ w_{+}, & x>0 .\end{cases}
$$

If $w_{-}<w_{+}$, then the problem (2.1) has the centered rarefaction wave solution $w^{r}(x, t)=w^{r}(x / t)$ given by

$$
w^{r}(x, t)= \begin{cases}w_{-}, & x / t \leq w_{-} \\ x / t, & w_{-} \leq x / t \leq w_{+}, \\ w_{+}, & x / t \geq w_{+}\end{cases}
$$

To construct a smooth rarefaction wave solution of the Burgers equation which approximates the centered rarefaction wave, we set for $\delta>0$,

$$
w_{\delta}(x)=w(\delta x)=\frac{w_{+}+w_{-}}{2}+\frac{\left(w_{+}-w_{-}\right)}{2} \tanh (\delta x)
$$

and for each $\delta>0$, we solve the following initial value problem

$$
\left\{\begin{array}{l}
w_{t}+\left(\frac{w^{2}}{2}\right)_{x}=0 \\
w(x, 0)=w_{\delta}(x) .
\end{array}\right.
$$

Next, we state certain properties that will be used later (see [30, 22] for a proof).

LEMma 2.1. For each $\delta>0$, the problem (2.2) has a unique global smooth solution $w_{\delta}^{r}(x, t)$, such that

(i) $w_{-}<w_{\delta}^{r}(x, t)<w_{+}, \partial_{x} w_{\delta}^{r}(x, t)>0$ for $x \in \mathbb{R}, t \geq 0, \delta>0$.

(ii) For any $1 \leq p \leq \infty$, there is a constant $C(p)$ depending only on $p$, such that

$$
\begin{aligned}
& \left\|\partial_{x} w_{\delta}^{r}(\cdot, t)\right\|_{L^{p}} \leq C(p) \min \left\{\left(w_{+}-w_{-}\right) \delta^{1-1 / p},\left(w_{+}-w_{-}\right)^{1 / p} t^{-1+1 / p}\right\}, \\
& \left\|\partial_{x}^{2} w_{\delta}^{r}(\cdot, t)\right\|_{L^{p}} \leq C(p) \min \left\{\left(w_{+}-w_{-}\right) \delta^{2-1 / p}, \delta^{1-1 / p} \frac{1}{t}\right\}, \\
& \left\|\partial_{x}^{3} w_{\delta}^{r}(\cdot, t)\right\|_{L^{p}} \leq C(p) \min \left\{\left(w_{+}-w_{-}\right) \delta^{3-1 / p}, \delta^{2-1 / p} \frac{1}{t}\right\} .
\end{aligned}
$$

(iii)

$$
\lim _{t \rightarrow+\infty} \sup _{x \in \mathbb{R}}\left|w_{\delta}^{r}(x, t)-w^{r}(x, t)\right|=0 .
$$

Now, set $w_{ \pm}=\lambda_{1}\left(v_{ \pm}, \bar{s}\right)$, and we define $V(x, t), U(x, t), S(x, t), \Theta(x, t)$, the smooth approximation of $\left(v^{r}, u^{r}, s^{r}, \theta^{r}\right)$, by

$$
\begin{aligned}
& \lambda_{1}(V(x, t), \bar{s})=w_{\delta}^{r}(x, t), \quad U(x, t)=u_{ \pm}+\int_{v_{ \pm}}^{V(x, t)} \sqrt{-p_{v}(z, \bar{s})} d z \\
& S(x, t)=\bar{s}, \quad \Theta(x, t)=\theta(V(x, t), \bar{s}) .
\end{aligned}
$$


Then, it is not difficult to see that $V(x, t), U(x, t), S(x, t), \Theta(x, t)$ satisfy

$$
\left\{\begin{array}{l}
V_{t}-U_{x}=0 \\
U_{t}+p(V, \Theta)_{x}=0 \\
S_{t}(V, \Theta)=0 \\
\Theta_{t}+\frac{\Theta p_{\theta}(V, \Theta)}{e_{\theta}} U_{x}=0
\end{array}\right.
$$

and due to Lemma 2.1, the following lemma holds for $V, U, S, \Theta$.

LEMma 2.2. The functions $V(x, t), U(x, t), S(x, t)$ and $\Theta(x, t)$ constructed above satisfy:

(i) $V_{t}=U_{x}>0$ for all $x \in \mathbb{R}, t \geq 0$.

(ii) For any $1 \leq p \leq \infty$, there is a positive constant $C(p)$ depending only on $p$, such that

$$
\begin{aligned}
& \left\|\left(V_{x}, U_{x}, \Theta_{x}\right)(\cdot, t)\right\|_{L^{p}} \leq C(p) \min \left\{\alpha \delta^{1-1 / p}, \alpha^{1 / p} t^{-1+1 / p}\right\} \\
& \left\|\left(V_{x x}, U_{x x}, \Theta_{x x}\right)(\cdot, t)\right\|_{L^{p}} \leq C(p) \min \left\{\alpha \delta^{2-1 / p}, \delta^{1-1 / p} t^{-1}\right\}, \\
& \left\|\left(V_{x x x}, U_{x x x}, \Theta_{x x x}\right)(\cdot, t)\right\|_{L^{p}} \leq C(p) \min \left\{\alpha \delta^{3-1 / p}, \delta^{2-1 / p} t^{-1}\right\} .
\end{aligned}
$$

(iii)

$$
\lim _{t \rightarrow \infty} \sup _{x \in \mathbb{R}}\left|(V, U, S, \Theta)(t, x)-\left(v^{r}, u^{r}, s^{r}, \theta^{r}\right)(t, x)\right|=0 .
$$

(iv)

$$
\left|\left(V_{t}, U_{t}, \Theta_{t}\right)(x, t)\right| \leq C\left|\left(V_{x}, U_{x}, \Theta_{x}\right)(x, t)\right|
$$

Consequently, from Lemmas 2.1 and 2.2, it follows that $(U, V, \Theta)(x, t)$ converges to $\left(u^{r}, v^{r}, \theta^{r}\right)(x, t)$ as $t \rightarrow \infty$.

Now, we reformulate the problem by a natural scaling. Due to the scale invariance of the Riemann problem (1.13), (1.16), we rescale the Cauchy problem $(1.1)_{1},(1.1)_{2}$ and (1.10) by

$$
y=x / \epsilon, \quad \tau=t / \epsilon, \quad \epsilon>0
$$

to obtain

$$
\left\{\begin{array}{l}
v_{\tau}-u_{y}=0, \\
u_{\tau}+p_{y}(v, \theta)=\left(\frac{u_{y}}{v}\right)_{y} \\
\theta_{\tau}+\frac{\theta p_{\theta}(v, \theta)}{e_{\theta}(v, \theta)} u_{y}=\frac{\mu}{e_{\theta}(v, \theta)}\left(\frac{\theta_{y}}{v}\right)_{y}+\frac{1}{e_{\theta}(v, \theta)} \frac{u_{y}^{2}}{v}
\end{array}\right.
$$

with initial data

$$
(u, v, \theta)(y, 0)=\left(u_{0}, v_{0}, \theta_{0}\right)(y), \quad y \in \mathbb{R}
$$

where $\mu=\kappa / \epsilon$ and by virtue of the assumptions (1.17),

$$
\underline{\mu} \leq \mu \leq \bar{\mu} \text { uniformly in } \epsilon>0 \text { for some positive constants } \underline{\mu}, \bar{\mu} \text {. }
$$


And in the case of the rarefaction wave initial data (1.16), the initial data (2.5) are

$$
\left(u_{0}, v_{0}, \theta_{0}\right)(y)= \begin{cases}\left(u_{-}, v_{-}, \theta_{-}\right), & x<0 \\ \left(u_{+}, v_{+}, \theta_{+}\right), & x>0\end{cases}
$$

If there exists a unique global solution $(u, v, \theta)(y, \tau)$ to the problem $(2.4),(2.5)$ with the same regularity as stated in Theorem 1.1, then the solution $\left(u^{\epsilon}, v^{\epsilon}, \theta^{\epsilon}\right)(x, t)$ to the problem $(1.1)_{1},(1.1)_{2},(1.10)$ and (1.12) is given by

$$
\left(u^{\epsilon}, v^{\epsilon}, \theta^{\epsilon}\right)(x, t)=(u, v, \theta)(x / \epsilon, t / \epsilon) .
$$

Hence, it follows that Theorem 1.1 can be proved if one can show

$$
\lim _{\tau \rightarrow \infty} \sup _{y \in \mathbb{R}}\left|(u, v, \theta)(y, \tau)-\left(u^{r}, v^{r}, \theta^{r}\right)\left(\frac{y}{\tau}\right)\right|=0,
$$

where $\left(u^{r}, v^{r}, \theta^{r}\right)(y / \tau)=\left(u^{r}, v^{r}, \theta^{r}\right)(x / t)$ is the centered 1-rarefaction wave solution defined by (1.15). Thus, the proof of Theorem 1.1 is reduced to showing that the centered rarefaction wave is a time-asymptotic state for the solution of (2.4) with discontinuous initial data (2.6), this will be a consequence of the following (more general) stability theorem.

THEOREM 2.3. Let $\left(u^{r}, v^{r}, \theta^{r}\right)(y / \tau)$ be the centered 1-rarefaction wave as in Theorem 1.1. Consider the Cauchy problem for (2.4), (2.5) where $\left(u_{0}, v_{0}, \theta_{0}\right)$ and its derivatives are sufficiently smooth away from $y=0$, but up to $y=0$ with a simple jump discontinuity at $y=0$. Assume that

$$
\left(u_{0}-u_{ \pm}, v_{0}-v_{ \pm}, \theta_{0}-\theta_{ \pm}\right) \in L^{2}\left(\mathbb{R}^{ \pm}\right), \quad v_{0 y} \in L^{2}\left(\mathbb{R}^{-}\right) \cap L^{2}\left(\mathbb{R}^{+}\right) .
$$

Then, there is a positive constant $\eta_{0}$, such that if

$$
\left\|\left(u_{0}-u_{ \pm}, v_{0}-v_{ \pm}, \theta_{0}-\theta_{ \pm}\right)\right\|_{L^{2}\left(\mathbb{R}^{ \pm}\right)}+\left\|v_{0 y}\right\|_{ \pm}+\alpha \leq \eta_{0}
$$

then the Cauchy problem (2.4), (2.5) has a unique global solution $(u, v, \theta)(y, \tau)$ in the same function class as in Theorem 1.1. Moreover,

$$
\lim _{\tau \rightarrow \infty} \sup _{y \in \mathbb{R}}\left|(u, v, \theta)(y, \tau)-\left(u^{r}, v^{r}, \theta^{r}\right)\left(\frac{y}{\tau}\right)\right|=0 .
$$

Theorem 2.3 looks like nonlinear stability of centered rarefaction waves for the compressible Navier-Stokes equations, see, e.g., [16, 20, 21, 19, 22]. The main difference is that for the nonlinear stability of centered rarefaction waves, initial perturbation is smooth, while here one has to deal with initial perturbation with discontinuities, the time evolution of which has to be controlled properly. But, some ideas from the study of nonlinear stability can be borrowed here.

The proof of Theorem 2.3 is broken up into several steps. We start with the observation that by making use of the smooth rarefaction wave $(U, V, \Theta)(y, \tau)$ constructed above (e.g. one may take $\delta=1$ ), one can decompose the solution $(u, v, \theta)(y, \tau)$ of (2.4), (2.5) into

$$
(\varphi, \psi, \phi)(y, \tau)=(v-V, u-U, \theta-\Theta)(y, \tau), \quad \xi(y, \tau)=s(y, \tau)-\bar{s} .
$$


Substituting the above decomposition into $(2.4),(2.5)$, we obtain the system for the functions $\varphi, \psi, \phi, \xi$ :

$$
(2.9)\left\{\begin{array}{l}
\varphi_{\tau}-\psi_{y}=0, \\
\psi_{\tau}+(p(v, \theta)-p(V, \Theta))_{y}=\left(\frac{u_{y}}{v}\right)_{y} \\
\phi_{\tau}+\frac{\theta p_{\theta}(v, \theta)}{e_{\theta}(v, \theta)} \psi_{y}+\left(\frac{\theta p_{\theta}(v, \theta)}{e_{\theta}(v, \theta)}-\frac{\Theta p_{\theta}(V, \Theta)}{e_{\theta}(V, \Theta)}\right) U_{y}=\frac{1}{e_{\theta}(v, \theta)}\left(\mu\left(\frac{\theta_{y}}{v}\right)_{y}+\frac{u_{y}^{2}}{v}\right), \\
\xi_{\tau}=\mu\left(\frac{\theta_{y}}{v \theta}\right)_{y}+\mu \frac{\theta_{y}^{2}}{v \theta^{2}}+\frac{u_{y}^{2}}{v \theta}
\end{array}\right.
$$

with initial data

$$
(\varphi, \psi, \phi, \xi)(y, 0)=\left(\varphi_{0}, \psi_{0}, \phi_{0}, \xi_{0}\right) \equiv\left(v_{0}-V_{0}, u_{0}-U_{0}, \theta_{0}-\Theta_{0}, s_{0}-\bar{s}\right)
$$

where $\left(\varphi_{0}, \psi_{0}, \phi_{0}, \xi_{0}\right)$ and its derivatives are sufficiently smooth away from $y=0$ but up to $y=0$, and $\left(\varphi_{0}, \psi_{0}, \phi_{0}, \xi_{0}\right) \in L^{2}(\mathbb{R}), \varphi_{0 y} \in L^{2}\left(\mathbb{R}^{-}\right) \cap L^{2}\left(\mathbb{R}^{+}\right)$.

We shall show that the Cauchy problem (2.9), (2.10) possesses a unique global solution $(\varphi, \psi, \phi, \xi)(y, \tau)$ in the same function class as for $\left(u^{\epsilon}, v^{\epsilon}, \theta^{\epsilon}\right)$ in Theorem 1.1. Moreover, $(\varphi, \psi, \phi)$ goes to zero uniformly as $\tau \rightarrow \infty$. This convergence then yields Theorem 2.3 due to Lemmas 2.1 and 2.2.

Proposition 2.4. There exists a positive constant $\eta_{0}$, such that if

$$
N^{2}(0):=\left\|\left(\varphi_{0}, \psi_{0}, \phi_{0}\right)\right\|^{2}+\left\|\varphi_{0 y}\right\|_{ \pm}^{2}+\alpha \leq \eta_{0}
$$

then the Cauchy problem (2.9), (2.10) has a unique global solution $(\varphi, \psi, \phi)(y, \tau)$ in the same function class as for $\left(u^{\epsilon}, v^{\epsilon}, \theta^{\epsilon}\right)(x, t)$ in Theorem 1.1, satisfying

(i)

$$
\sup _{\tau \geq 0}\left(\|(\varphi, \psi, \phi)(\tau)\|^{2}+\left\|\varphi_{y}(\tau)\right\|_{ \pm}^{2}\right)+\int_{0}^{\infty}\left\|\left(\varphi_{y}, \psi_{y}, \phi_{y}\right)(\tau)\right\|_{ \pm}^{2} d \tau \leq C\left\{N^{2}(0)+\delta^{1 / 4}\right\}
$$

(ii) For any $\tau_{0}>0$, there is a constant $C\left(\tau_{0}\right)>0$, such that

$$
\begin{aligned}
\sup _{\tau \geq \tau_{0}}\left(\left\|\left(\psi_{y}, \phi_{y}\right)(\tau)\right\|_{ \pm}^{2}+\left\|\left(\psi_{y y}, \phi_{y y}\right)(\tau)\right\|_{ \pm}^{2}\right) & +\int_{\tau_{0}}^{\infty}\left\|\left(\psi_{y y}, \psi_{y \tau}, \phi_{y y}, \phi_{y \tau}\right)(\tau)\right\|_{ \pm}^{2} d \tau \\
& \leq C\left(\tau_{0}\right)\left\{N^{2}(0)+\delta^{1 / 4}\right\} .
\end{aligned}
$$

(iii) The jump discontinuity of $\varphi(y, \tau)$ at $y=0$ is bounded from above by

$$
|[\varphi](\tau)| \leq C_{1} \exp \left\{-C_{2} \tau\right\}, \quad \forall \tau \in(0, \infty) .
$$

Here $C, C_{1}, C_{2}$ are positive constants independent of $\tau$.

REMARK 2.1. It is not difficult to see that for the rarefaction wave initial data (1.16), the smallness of $\left\|\left(\varphi_{0}, \psi_{0}, \phi_{0}\right)\right\|$ in the condition (2.11) is satisfied provided that $\delta$ is appropriately large but without smallness of $\alpha$, while the smallness of $\left\|\varphi_{0 y}\right\|_{ \pm}$holds provided that for fixed $\delta, \alpha$ is small enough.

Proof. To show Proposition 2.4, we combine the local existence and regularity result in [13] with an a priori energy estimate based on the nature of the underlying rarefaction wave. Firstly, we state the following local existence, the proof of which can be found in [13]. 
LEMma 2.5. (Hoff [13]) Suppose that $N(0)$ is suitably small so that there exist two positive constants $\underline{v}$ and $\bar{v}$ with $\underline{v} \leq v_{0}^{1}(y) \leq \bar{v}$ for all $y \in \mathbb{R}$. Then, there is $a$ constant $T>0$, such that the Cauchy problem (2.9), (2.10) has a solution $(\varphi, \psi, \phi)$ on $\mathbb{R} \times[0, T]$ in the same function class as for $\left(u^{\epsilon}, v^{\epsilon}, \theta^{\epsilon}\right)$ in Theorem 1.1. Moreover, $\varphi, \psi, \phi$ satisfy

(i) There exists a positive constant $C$, such that

$$
\sup _{\tau \geq 0}\left(\|(\varphi, \psi, \phi)(\tau)\|^{2}+\left\|\varphi_{y}(\tau)\right\|_{ \pm}^{2}\right)+\int_{0}^{T}\left\|\left(\varphi_{y}, \psi_{y}, \phi_{y}\right)(\tau)\right\|_{ \pm}^{2} d \tau \leq C\left\{N^{2}(0)+\delta^{1 / 4}\right\} .
$$

(ii) There is a positive constant $C$, such that

$$
\begin{aligned}
\sup _{0 \leq \tau \leq T}\left(\left\|\left(\psi_{y}, \phi_{y}\right)(\tau)\right\|_{ \pm}^{2}+\left\|\left(\psi_{y y}, \phi_{y y}\right)(\tau)\right\|_{ \pm}^{2}\right) & +\int_{\tau_{0}}^{T}\left\|\left(\psi_{y y}, \psi_{y \tau}, \phi_{y y}, \phi_{y \tau}\right)(\tau)\right\|_{ \pm}^{2} d \tau \\
& \leq C\left\{N^{2}(0)+\delta^{1 / 4}\right\} .
\end{aligned}
$$

(iii) There are constants $C_{1}, C_{2}>0$ independent of $T$, such that

$$
|[p(v, e)]|=\left|\left[\frac{u_{y}}{v}\right]\right| \leq C_{1} \exp \left\{-C_{2} \tau\right\}
$$

By virtue of Lemma 2.5 and the continuation in time of the local solution, we see that to complete the proof of Proposition 2.4, it suffices to prove the following a priori estimate, the proof of which will be postponed to the next section.

Proposition 2.6. (A priori estimate) Let the assumptions in Lemma 2.5 be satisfied. Assume that the Cauchy problem (2.9), (2.10) has a solution $(\varphi, \psi, \phi)(y, \tau)$ on $\mathbb{R} \times[0, \tau]$ for some $\tau>0$ in the same function class as in Lemma 2.5. Denote

$$
N^{2}\left(\tau_{0}, \tau\right):=\sup _{\tau_{0} \leq s \leq \tau}\left\{\|(\varphi, \psi, \phi)(\tau)\|^{2}+\left\|\varphi_{y}(\tau)\right\|_{ \pm}^{2}\right\}, \quad 0 \leq \tau_{0} \leq \tau .
$$

Then, there are positive constants $\eta_{1}$ and $C$ independent of $\tau_{1}$, such that for each fixed $\tau_{0}$, if

$$
N^{2}\left(\tau_{0}, \tau_{1}\right) \leq \eta_{1}
$$

then the following estimates hold

$$
\begin{gathered}
N^{2}\left(\tau_{0}, \tau_{1}\right)+\int_{\tau_{0}}^{\tau_{1}}\left\|\left(\varphi_{y}, \psi_{y}, \phi_{y}\right)(\tau)\right\|_{ \pm}^{2} d \tau \leq C\left\{\|(\varphi, \psi, \phi)\left(\tau_{0}\left\|^{2}+\right\| \varphi_{y}\left(\tau_{0}\right) \|_{ \pm}^{2}+\delta^{1 / 4}\right\},\right. \\
\sup _{0 \leq \tau \leq T}\left\|\left(\psi_{y}, \phi_{y}\right)(\tau)\right\|_{ \pm}^{2}+\int_{\tau_{0}}^{\tau_{1}}\left\|\left(\psi_{y y}, \phi_{y y}\right)\right\|_{ \pm}^{2} d \tau \\
\leq C\left\{\left\|(\varphi, \psi, \phi)\left(\tau_{0}\right)\right\|_{ \pm}^{2}+\left\|\left(\varphi_{y}, \psi_{y}, \phi_{y}\right)\left(\tau_{0}\right)\right\|_{ \pm}^{2}+\delta^{1 / 4}\right\} .
\end{gathered}
$$

Proof of Theorem 2.3. By the systems (2.3) and (2.9), Lemma 2.2, CauchySchwarz's and Sobolev's inequalities, we easily find that

$$
\int_{0}^{\infty}\left\|\left(\varphi_{t}, \psi_{t}, \phi_{t}\right)(\tau)\right\|_{L^{\infty}}^{2} d \tau<\infty
$$

which together with Proposition 2.4 yields $\lim _{\tau \rightarrow \infty} \sup _{y}|(\varphi(y, \tau), \psi(y, \tau), \phi(y, \tau))| \rightarrow$ 0. Hence, In view of Lemma 2.2, we have proved Theorem 2.3. 
3. Uniform a priori estimates. In this section we derive the key a priori estimates given in Proposition 2.6. First, we introduce the normalized entropy $\eta(v, u, s, V, U, S)$ around $(V, U, S)$ :

$$
\begin{aligned}
\eta(v, u, s, V, U, S):= & \left(e(v, \theta)+\frac{u^{2}}{2}\right)-\left(e(V, \Theta)+\frac{U^{2}}{2}\right) \\
& -\{-p(V, \Theta)(v-V)+U(u-U)+\Theta(s-S)\},
\end{aligned}
$$

where we have used the fact that $e_{v}(v, s)=-p(v, \theta), e_{s}(v, s)=0$.

An easy computation implies that $\eta$ satisfies the equation:

$$
\begin{aligned}
\eta_{\tau}(v, u, s, V, U, S)+ & \{(p(v, \theta)-p(V, \Theta) \psi)\}_{y}+\left(\Theta \frac{\psi_{y}^{2}}{v \theta}+\mu \Theta \frac{\phi_{y}^{2}}{v \theta^{2}}\right) \\
& +\left\{p(v, s)-p(V, \bar{s})-p_{v}(V, \bar{s}) \psi-p_{s}(V, \bar{s}) \xi\right\} U_{y} \\
& =\left(\frac{\psi \psi_{y}}{v}+\mu \frac{\phi \phi_{y}}{v \theta}\right)_{y}+\left(-\frac{U_{y} \psi \phi_{y}}{v^{2}}+2 \frac{U_{y} \phi \psi_{y}}{v \theta}-\mu \frac{\Theta \phi \varphi_{y}}{v^{2} \theta}+\mu \frac{\Theta_{y} \phi \phi_{y}}{v \theta^{2}}\right) \\
& +\left(\frac{U_{x} x \psi}{v}+\mu \frac{\Theta_{y y} \phi}{v \theta}\right)+\left(-\frac{V_{y} U_{y} \psi}{v^{2}}+\frac{U_{y}^{2} \phi}{v \theta}-\mu \frac{V_{y} \Theta_{y} \phi}{v^{2} \theta}\right) .
\end{aligned}
$$

Employing (3.1), one has

Lemma 3.1. Suppose that the assumptions of Proposition 2.6 hold. Then,

$$
\begin{aligned}
& \|(\varphi, \psi, \phi)(\tau)\|^{2}+\int_{\tau_{0}}^{\tau}\left(\left\|\sqrt{V_{\tau}}(\varphi, \phi)(\hat{\tau})\right\|^{2}+\left\|\left(\psi_{y}, \phi_{y}\right)(\hat{\tau})\right\|_{ \pm}^{2}\right) d \hat{\tau} \\
& \leq C\left\{\left\|(\varphi, \psi, \phi)\left(\tau_{0}\right)\right\|^{2}+\delta^{1 / 4}+N\left(\tau_{0}, \tau\right)^{2 / 3} \int_{\tau_{0}}^{\tau}\left\|\varphi_{y}(\hat{\tau})\right\|_{ \pm}^{2} d \hat{\tau}\right\} .
\end{aligned}
$$

Proof. Integrating (3.1) with respect to $\tau$ and $y$, we get

$\|(\varphi, \psi, \phi)(\tau)\|^{2}+\int_{\tau_{0}}^{\tau}\left(\left\|\sqrt{V_{\tau}}(\varphi, \phi)\right\|^{2}+\left\|\left(\psi_{y}, \phi_{y}\right)\right\|_{ \pm}^{2}\right)(\hat{\tau}) d \hat{\tau} \leq C\left\{\left\|(\varphi, \psi, \phi)\left(\tau_{0}\right)\right\|^{2}+\sum_{j=1}^{j=4} R_{j}\right\}$,

where

$$
\begin{aligned}
& R_{1}=\int_{\tau_{0}}^{\tau} \int_{ \pm}\left\{-(p(v, \theta)-p(V, \Theta) \psi)_{y}+\left(\frac{\psi \psi_{y}}{v}+\mu \frac{\phi \phi_{y}}{v \theta}\right)_{y}\right\}(y, \hat{\tau}) d y d \hat{\tau} \\
& R_{2}=\int_{\tau_{0}}^{\tau} \int_{ \pm}\left(\left|\psi U_{y y}\right|+\left|\phi \Theta_{y y}\right|\right)(y, \hat{\tau}) d y d \hat{\tau} \\
& R_{3}=\int_{\tau_{0}}^{\tau} \int_{ \pm}\left(\left|U_{y} \psi \varphi_{y}\right|+\left|U_{y} \psi_{y} \phi\right|+\left|\Theta_{y} \varphi_{y} \phi\right|+\left|\phi \phi_{y} \Theta_{y}\right|\right)(y, \hat{\tau}) d y d \hat{\tau} \\
& R_{4}=\int_{\tau_{0}}^{\tau} \int_{ \pm}\left(\left|V_{y} U_{y} \psi\right|+\left|U_{y}^{2} \phi\right|+\left|\phi \Theta_{y} V_{y}\right|\right)(y, \hat{\tau}) d y d \hat{\tau} .
\end{aligned}
$$

Here we have used the assumption (1.17), the smallness of $N\left(\tau_{0}, \tau\right)$ such that $\underline{v} \leq v \leq \bar{v}$ and $\underline{\theta} \leq \theta \leq \bar{\theta}$ for some positive constants $\underline{v}, \bar{v}, \underline{\theta}, \bar{\theta}$, the convexity of $p(v, s)$ with respect to $v$ and $s$, and the equivalence of $|(\varphi, \psi, \xi)|^{2}$ to $|(\varphi, \psi, \phi)|^{2}$.

Recalling the definition of $N\left(\tau, \tau_{0}\right)$ and applying Lemma 2.2, for given $\alpha, R_{j}$ can be estimated as follows.

$$
R_{1}=\int_{\tau_{0}}^{\tau}\left\{\left[-p(v, \theta)+p(V, \Theta)+\frac{\psi_{y}}{v}\right] \psi+\left[\frac{\phi \phi_{y}}{v \theta}\right]\right\}(\hat{\tau}) d \hat{\tau}=0
$$




$$
\begin{gathered}
R_{2} \leq C \int_{\tau_{0}}^{\tau}\|(\psi, \phi)(\hat{\tau})\|^{1 / 2}\left\|\left(\psi_{y}, \phi_{y}\right)(\hat{\tau})\right\|^{1 / 2}\left\|U_{y y}(\hat{\tau})\right\|_{L^{1}} d \hat{\tau} \\
\leq C \int_{\tau_{0}}^{\tau}\left\{N\left(\tau_{0}, \tau\right)\left\|\left(\phi_{y}, \psi_{y}\right)(\hat{\tau})\right\|^{2}+\left\|U_{y y}(\hat{\tau})\right\|_{L^{1}}^{4 / 3}\right\} d \hat{\tau} \\
\leq C\left\{N\left(\tau_{0}, \tau\right) \int_{\tau_{0}}^{\tau}\left\|\left(\phi_{y}, \psi_{y}\right)(\hat{\tau})\right\|^{2} d \hat{\tau}+\delta^{1 / 4}\right\}, \\
R_{4} \leq C \int_{\tau_{0}}^{\tau}\|(\psi, \phi)(\hat{\tau})\|^{1 / 2}\left\|\left(\psi_{y}, \phi_{y}\right)(\hat{\tau})\right\|^{1 / 2}\left\|U_{y}(\hat{\tau})\right\|^{2} d \hat{\tau} \\
\leq C \int_{\tau_{0}}^{\tau}\left\{N\left(\tau_{0}, \tau\right)\left\|\left(\phi_{y}, \psi_{y}\right)(\hat{\tau})\right\|^{2}+\left\|U_{y}(\hat{\tau})\right\|^{8 / 3}\right\} d \hat{\tau} \\
\leq C\left\{N\left(\tau_{0}, \tau\right) \int_{\tau_{0}}^{\tau}\left\|\left(\phi_{y}, \psi_{y}\right)(\hat{\tau})\right\|^{2} d \hat{\tau}+\delta^{1 / 4}\right\}
\end{gathered}
$$

and

$$
\begin{aligned}
R_{3} & \leq C \int_{\tau_{0}}^{\tau}\|(\psi, \phi, \varphi)(\hat{\tau})\|^{1 / 2}\left\|\left(\psi_{y}, \phi_{y}, \varphi_{y}\right)(\hat{\tau})\right\|^{3 / 2}\left\|U_{y}(\hat{\tau})\right\| d \hat{\tau} \\
& \leq C \int_{\tau_{0}}^{\tau}\left\{N\left(\tau_{0}, \tau\right)^{2 / 3}\left\|\left(\phi_{y}, \psi_{y}, \varphi_{y}\right)(\hat{\tau})\right\|^{2}+\left\|U_{y}(\hat{\tau})\right\|^{4}\right\} d \hat{\tau} \\
& \leq C\left\{N\left(\tau_{0}, \tau\right)^{2 / 3} \int_{\tau_{0}}^{\tau}\left\|\left(\phi_{y}, \psi_{y}\right)(\hat{\tau})\right\|^{2} d \hat{\tau}+\delta^{1 / 4}\right\}
\end{aligned}
$$

where we have used Sobolev's inequality and the following inequality:

$$
\begin{aligned}
\int_{\tau_{0}}^{\tau}\left\|\partial_{x}^{i} U(\hat{\tau})\right\|_{L^{p}}^{a+b} d \hat{\tau} & \leq \sup _{r \in\left[\tau_{0}, \tau\right]}\left\|\partial_{x}^{i} U(\hat{\tau})\right\|_{L^{p}}^{a} \int_{\tau_{0}}^{\tau}\left\|\partial_{x}^{i} U(\hat{\tau})\right\|_{L^{p}}^{b} d \hat{\tau} \\
& \leq C \delta^{(i-1 / p) a} \int_{\tau_{0}}^{\tau}\left\|\partial_{x}^{i} U(\hat{\tau})\right\|_{L^{p}}^{b} d \hat{\tau} .
\end{aligned}
$$

Substituting the above estimates for $R_{j}(j=1, \cdots, 4)$ into (3.3), we obtain (3.2). This completes the proof.

We now proceed to derive bounds for the term $\int_{\tau_{0}}^{\tau}\left\|\varphi_{x}(\hat{\tau})\right\|_{ \pm}^{2} d \hat{\tau}$, and we have

Lemma 3.2. Suppose that the assumptions of Proposition 2.6 hold. Then,

$$
\begin{aligned}
\left\|\varphi_{y}(\tau)\right\|_{ \pm}^{2} & +\int_{\tau_{0}}^{\tau}\left\|\varphi_{y}(\hat{\tau})\right\|_{ \pm}^{2} d \hat{\tau} \leq C\left\{\left\|\left(\varphi_{y}, \psi\right)\left(\tau_{0}\right)\right\|^{2}+\|\psi(\tau)\|_{ \pm}^{2}\right. \\
& \left.+\int_{\tau_{0}}^{\tau}\left(\left\|\left(\psi_{y}, \phi_{y}\right)(\hat{\tau})\right\|_{ \pm}^{2}+\left\|\sqrt{V_{\tau}(\hat{\tau})}(\varphi, \phi)(\hat{\tau})\right\|_{ \pm}^{2}\right) d \hat{\tau}+\delta\right\} .
\end{aligned}
$$

Proof. By (2.9), we easily find that

$$
\begin{aligned}
\left(\frac{1}{2}\left(\frac{\varphi_{y}}{v}\right)^{2}-\frac{\varphi_{y}}{v} \psi\right)_{\tau} & -p_{v}(v, \theta) \frac{\varphi_{y}^{2}}{v}-\left(\frac{\psi_{y}^{2}}{v}+\frac{p_{\theta} \varphi_{y} \phi_{y}}{v}\right)+\left(\frac{\psi \psi_{y}}{v}\right)_{y} \\
& =\left\{V_{y}\left(p_{v}(v, \theta)-p_{v}(V, \Theta)\right) \frac{\varphi_{y}}{v}+\Theta_{y}\left(p_{\theta}(v, \theta)-p_{\theta}(V, \Theta)\right) \frac{\varphi_{y}}{v}\right. \\
& \left.+\frac{U_{y} \psi \varphi_{y}}{v^{2}-V_{y} \psi \psi_{y} / v^{2}}\right\}+\frac{V_{y} \psi_{y} \varphi_{y}}{v^{3}}-\frac{U_{y y} \varphi_{y}}{v^{2}}+\frac{V_{y} U_{y} \varphi_{y}}{v^{3}} .
\end{aligned}
$$


Integrating (3.5) with respect to $y, \tau$ over $\left(\tau_{0}, \tau\right) \times \mathbb{R}$, we infer

$$
\begin{aligned}
\left\|\varphi_{y}(\tau)\right\|_{ \pm}^{2}+\int_{\tau_{0}}^{\tau}\left\|\varphi_{y}(\hat{\tau})\right\|_{ \pm}^{2} d \hat{\tau} \leq & C\left\{\left\|\left(\varphi_{y}, \psi\right)\left(\tau_{0}\right)\right\|^{2}+\|\psi(\tau)\|^{2}+\int_{\tau_{0}}^{\tau}\left(\left\|\left(\psi_{y}, \phi_{y}\right)\right\|_{ \pm}^{2}\right.\right. \\
& \left.\left.+\left\|\sqrt{V_{t}}(\varphi, \phi)\right\|_{ \pm}^{2}\right)(\hat{\tau}) d \hat{\tau}+\sum_{j=5}^{j=7} R_{j}\right\}
\end{aligned}
$$

with

$$
\begin{aligned}
& R_{5}=-\int_{\tau_{0}}^{\tau} \int_{ \pm}\left(\frac{\psi \psi_{y}}{v}\right)_{y} d \hat{\tau}, \quad R_{6}=\int_{\tau_{0}}^{\tau} \int_{ \pm}\left(\left|\psi U_{y} \varphi_{y}\right|+\left|V_{y} \psi \psi_{y}\right|\right)(y, \hat{\tau}) d y d \hat{\tau} \\
& R_{7}=\int_{\tau_{0}}^{\tau} \int_{ \pm}\left(\left|\psi_{y} V_{y} \varphi_{y}\right|+\left|U_{y y} \varphi_{y}\right|+\left|U_{y} V_{y} \varphi_{y}\right|\right)(y, \hat{\tau}) d y d \hat{\tau}
\end{aligned}
$$

where $R_{5}, R_{6}, R_{7}$ can be bounded as follows, using Sobolev's imbedding theorem and Lemma 2.5 (iii).

$$
\begin{aligned}
R_{5} & =\int_{\tau_{0}}^{\tau}\left[\frac{\psi \psi_{y}}{v}\right] d \hat{\tau}=\int_{\tau_{0}}^{\tau} \psi\left[\frac{\psi_{y}}{v}\right] d \hat{\tau} \leq \int_{\tau_{0}}^{\tau}\|\psi\|^{1 / 2}\left\|\psi_{y}\right\|_{ \pm}^{1 / 2}\left[\frac{u_{y}}{v}\right] d \hat{\tau} \\
& \leq \frac{1}{4} \int_{\tau_{0}}^{\tau}\left\|\psi_{y}\right\|_{ \pm}^{2} d \hat{\tau}+C \sup _{\tau_{0} \leq s \leq \tau}\|\psi(s)\|^{2 / 3}\left|\left[\varphi\left(\tau_{0}\right)\right]\right|^{4 / 3} \int_{\tau_{0}}^{\tau} \exp \left(-\frac{4}{3} C_{3}\left(\hat{\tau}-\tau_{0}\right)\right) d \hat{\tau} \\
& \leq \frac{1}{4} \int_{\tau_{0}}^{\tau}\left\|\psi_{y}\right\|_{ \pm}^{2} d \hat{\tau}+\frac{1}{3} \sup _{\tau_{0} \leq s \leq \tau}\|\psi(s)\|^{2}+C\left\{\|\left(\varphi\left(\tau_{0}\right)\left\|^{2}+\right\| \varphi_{y}\left(\tau_{0}\right) \|^{2}\right)\right\}, \\
R_{6} & \leq C \int_{\tau_{0}}^{\tau}\|\psi(\hat{\tau})\|^{1 / 2}\left\|\psi_{y}(\hat{\tau})\right\|^{1 / 2}\left\|U_{y}(\hat{\tau})\right\|\left\|\left(\varphi_{y}, \psi_{y}\right)(\hat{\tau})\right\| d \hat{\tau} \\
& \leq C\left\{N\left(\tau_{0}, \tau\right)^{2 / 3} \int_{\tau_{0}}^{\tau}\left\|\left(\varphi_{y}, \psi_{y}\right)(\hat{\tau})\right\|^{2} d \hat{\tau}+\int_{\tau_{0}}^{\tau}\left\|U_{y}(\hat{\tau})\right\|^{4} d \hat{\tau}\right\} \\
& \leq C\left\{N\left(\tau_{0}, \tau\right)^{2 / 3} \int_{\tau_{0}}^{\tau}\left\|\left(\varphi_{y}, \psi_{y}\right)(\hat{\tau})\right\|^{2} d \hat{\tau}+\delta^{1 / 4}\right\} \\
R_{7} & \leq C \alpha \int_{\tau_{0}}^{\tau}\left\|\varphi_{y}\right\|_{ \pm}\left\|\psi_{y}\right\|_{ \pm} d \hat{\tau}+\int_{\tau_{0}}^{\tau}\left(\left\|U_{y y}\right\|\left\|\varphi_{y}\right\|_{ \pm}+\left\|V_{y}\right\|_{L^{4}}\left\|U_{y}\right\|_{L^{4}}\left\|\varphi_{y}\right\|_{ \pm}\right) d \hat{\tau} \\
& \leq \frac{1}{2} \int_{\tau_{0}}^{\tau}\left\|\varphi_{y}(\hat{\tau})\right\|_{ \pm}^{2} d \hat{\tau}+C \int_{\tau_{0}}^{\tau}\left(\left\|\psi_{y}\right\|_{ \pm}^{2}+\left\|U_{y}\right\|_{L^{4}}^{4}+\left\|V_{y}\right\|_{L^{4}}^{4}+\left\|U_{y y}\right\|^{2}\right) d \hat{\tau} \\
& \leq \frac{1}{2} \int_{\tau_{0}}^{\tau}\left\|\varphi_{y}(\hat{\tau})\right\|^{2} d \hat{\tau}+C\left\{\int_{\tau_{0}}^{\tau}\left\|\psi_{y}(\hat{\tau})\right\|^{2} d \hat{\tau}+\delta^{1 / 4}\right\} .
\end{aligned}
$$

Inserting the estimates for $R_{j}(j=5,6,7)$ into (3.6), we arrive at

$$
\begin{aligned}
\left\|\varphi_{y}(\tau)\right\|_{ \pm}^{2} & +\int_{\tau_{0}}^{\tau}\left\|\varphi_{y}(\hat{\tau})\right\|_{ \pm}^{2} d \hat{\tau} \leq C\left\{\left\|\left(\varphi_{y}, \psi\right)\left(\tau_{0}\right)\right\|_{ \pm}^{2}+\|\psi(\tau)\|^{2}\right. \\
& \left.+\int_{\tau_{0}}^{\tau}\left(\left\|\left(\psi_{y}, \phi_{y}\right)(\hat{\tau})\right\|_{ \pm}^{2}+\left\|\sqrt{V_{\tau}}(\varphi, \phi)(\hat{\tau})\right\|^{2}\right) d \hat{\tau}+\delta^{1 / 4}\right\} .
\end{aligned}
$$

Finally, combining Lemma 3.1 with Lemma 3.2, we conclude

$$
\begin{aligned}
\left\|\left(\varphi, \psi, \phi, \varphi_{y}\right)(\tau)\right\|^{2} & +\int_{\tau_{0}}^{\tau}\left(\left\|\sqrt{V_{\tau}(\hat{\tau})}(\varphi, \phi)(\hat{\tau})\right\|^{2}+\left\|\left(\varphi_{y}, \psi_{y}, \phi_{y}\right)(\hat{\tau})\right\|_{ \pm}^{2}\right) d \hat{\tau} \\
& \leq C\left(\left\|(\varphi, \psi, \phi)\left(\tau_{0}\right)\right\|^{2}+\left\|\varphi_{y}\left(\tau_{0}\right)\right\|_{ \pm}^{2}+\delta^{1 / 4}\right) .
\end{aligned}
$$


Comparing with the standard energy estimate for the compressible Navier-Stokes equations, we refer (3.8) to the basic energy estimate.

Next, we proceed to estimate higher order derivatives of $\psi, \phi$ in the space $L^{\infty}\left(\tau_{0}, \tau ; L^{2}\left(\mathbb{R}^{ \pm}\right)\right)$.

Lemma 3.3. Suppose that the assumptions of Proposition 2.6 hold. Then,

$$
\left\|\psi_{y}(\tau)\right\|_{ \pm}^{2}+\int_{\tau_{0}}^{\tau}\left\|\psi_{y y}(\tau)\right\|_{ \pm}^{2} d \tau \leq C\left(\left\|\psi_{y}\left(\tau_{0}\right)\right\|_{ \pm}+\left\|(\varphi, \psi, \phi)\left(\tau_{0}\right)\right\|^{2}+\delta^{1 / 4}\right) .
$$

Proof. Multiplying the second equation of (2.9) by $-\psi_{y y}$, one obtains

$$
\begin{aligned}
\left(\frac{\psi_{y}^{2}}{2}\right)_{\tau} & +\frac{\psi_{y y}^{2}}{v}-\left(\psi_{\tau} \psi_{y}\right)_{y}=\left(p_{v}(v, \theta) \varphi_{y}+p_{\theta}(v, \theta) \phi_{y}\right) \psi_{y y}+\frac{\varphi_{y} \psi_{y} \psi_{y y}}{v^{2}} \\
& +V_{y}\left\{p_{v}(v, \theta)-p_{v}(V, \Theta)\right\} \psi_{y y}+\Theta_{y}\left\{p_{\theta}(v, \theta)-p_{\theta}(V, \Theta)\right\} \psi_{x x} \\
& +\frac{V_{y} \psi_{y} \psi_{y y}}{v^{2}}+\frac{U_{y} \varphi_{y} \psi_{y y}}{v^{2}}-\frac{U_{y y} \psi_{y y}}{v}+\frac{V_{y} U_{y} \psi_{y y}}{v^{2}}
\end{aligned}
$$

which, by integrating with respect to $y$ and $\tau$, leads to

$$
\begin{aligned}
& \left\|\psi_{y}(\tau)\right\|_{ \pm}^{2}+\int_{\tau_{0}}^{\tau}\left\|\psi_{y y}(\hat{\tau})\right\|_{ \pm}^{2} d \hat{\tau} \leq\left\|\psi_{y}\left(\tau_{0}\right)\right\|_{ \pm}^{2}+\int_{\tau_{0}}^{\tau} \int_{ \pm}\left(\psi_{\tau} \psi_{y}\right)_{y} d y d \hat{\tau} \\
& +C\left\{\int_{\tau_{0}}^{\tau}\left(\left\|\left(\varphi_{y}, \psi_{y}, \phi_{y}\right)(\hat{\tau})\right\|_{ \pm}^{2}+\left\|\sqrt{V_{t}}(\varphi, \phi)(\hat{\tau})\right\|^{2}\right) d \hat{\tau}\right. \\
& +\int_{\tau_{0}}^{\tau} \int_{ \pm}\left(\left|\varphi_{y} \psi_{y} \psi_{y y}\right|+\left|V_{y} \psi_{y} \psi_{y y}\right|+\left|U_{y} \varphi_{y} \psi_{y y}\right|\right. \\
& \left.\left.+\left|\psi_{y y}\right|\left(\left|U_{y y}\right|+\left|U_{y}^{2}\right|\right)\right) d y d \hat{\tau}\right\} .
\end{aligned}
$$

The terms on the right hand side of (3.11) can be bounded as follows,

$$
\begin{aligned}
\int_{\tau_{0}}^{\tau} \int_{ \pm}\left(\psi_{\tau} \psi_{y}\right)_{y} d y d \hat{\tau} & =\left.\left[\psi \psi_{y}\right]\right|_{\tau_{0}} ^{\tau}-\int_{\tau_{0}}^{\tau} \psi\left[\psi_{y}\right]_{\tau} d \hat{\tau}=\left.\psi\left[\psi_{y}\right]\right|_{\tau_{0}} ^{\tau}-\int_{\tau_{0}}^{\tau} \psi\left[\psi_{y}\right]_{\tau} d \hat{\tau} \\
& \leq \frac{1}{8} \int_{\tau_{0}}^{\tau}\left\|\psi_{y y}(\hat{\tau})\right\|_{ \pm}^{2} d \hat{\tau}+C \int_{\tau_{0}}^{\tau}\left(\|\varphi\|^{2}+\|\psi\|^{2}+\left\|\psi_{y}\right\|_{ \pm}^{2}\right)(\hat{\tau}) d \hat{\tau}
\end{aligned}
$$

where we have used the fact that the jump $\left[u_{y}\right]$ decays exponentially in $\tau$ (cf. the estimate of $R_{5}$ in the proof of Lemma 3.2); and

$$
\begin{aligned}
& \int_{\tau_{0}}^{\tau} \int_{ \pm}\left|\left(\varphi_{y} \psi_{y} \psi_{y y}\right)(y, \hat{\tau})\right| d x d \hat{\tau} \leq C \int_{\tau_{0}}^{\tau}\left\|\varphi_{y}(\hat{\tau})\right\|_{ \pm}\left\|\psi_{y}(\hat{\tau})\right\|_{ \pm}^{1 / 2}\left\|\psi_{y y}(\cdot, \hat{\tau})\right\|_{ \pm}^{3 / 2} d \hat{\tau} \\
& \leq \frac{1}{8} \int_{\tau_{0}}^{\tau}\left\|\psi_{y y}(\hat{\tau})\right\|_{ \pm}^{2} d \hat{\tau}+C N\left(\tau_{0}, \tau\right) \int_{\tau_{0}}^{\tau}\left\|\psi_{y}(\hat{\tau})\right\|_{ \pm}^{2} d \hat{\tau} \\
& \int_{\tau_{0}}^{\tau} \int_{ \pm}\left|\psi_{y y}(y, \hat{\tau})\right|\left(\left|V_{y} \psi_{y}\right|+\left|U_{y} \varphi_{y}\right|\right)(y, \hat{\tau}) d y d \hat{\tau} \\
& \leq \frac{1}{8} \int_{\tau_{0}}^{\tau}\left\|\psi_{y y}(\hat{\tau})\right\|_{ \pm}^{2} d \hat{\tau}+C \delta \int_{\tau_{0}}^{\tau}\left\|\left(\varphi_{y}, \psi_{y}\right)(\hat{\tau})\right\|_{ \pm} d \hat{\tau}
\end{aligned}
$$

and

$$
\int_{\tau_{0}}^{\tau} \int_{ \pm}\left|\left(\psi_{y y}(y, \tau)\right)\right|\left(\left|U_{y y}\right|+\left|U_{y}\right|^{2}\right)(y, \tau) d y d \tau
$$




$$
\begin{aligned}
& \leq \frac{1}{8} \int_{\tau_{0}}^{\tau}\left\|\psi_{y y}(\hat{\tau})\right\|_{ \pm}^{2} d \hat{\tau}+C \int_{\tau_{0}}^{\tau}\left(\left\|U_{y y}\right\|^{2}+\left\|U_{y}\right\|_{L^{4}}^{4}\right)(\hat{\tau}) d \hat{\tau} \\
& \leq \frac{1}{8} \int_{\tau_{0}}^{\tau}\left\|\psi_{y y}(\hat{\tau})\right\|_{ \pm}^{2} d \hat{\tau}+C \delta^{1 / 4} .
\end{aligned}
$$

Substituting the above estimates into (3.10), we obtain (3.9).

Similarly, we can bound the derivatives of $\phi$ as follows.

Lemma 3.4. Assume that the assumptions of Proposition 2.6 hold. Then,

$$
\left\|\phi_{y}(\tau)\right\|_{ \pm}^{2}+\int_{\tau_{0}}^{\tau}\left\|\phi_{y y}(\hat{\tau})\right\|_{ \pm}^{2} d \hat{\tau} \leq C\left(\left\|(\varphi, \psi, \phi)\left(\tau_{0}\right)\right\|^{2}+\left\|\phi_{y}\left(\tau_{0}\right)\right\|_{ \pm}^{2}+\delta^{1 / 4}\right) .
$$

Proof. Multiplying the third equation of $(2.9)$ by $-\phi_{y y}$, then integrating with respect to $y$ and $\tau$, and utilizing (3.7) and (3.8), we deduce that

$$
\begin{aligned}
& \left\|\phi_{y}(\tau)\right\|^{2}+\int_{\tau_{0}}^{\tau}\left\|\phi_{y y}(\hat{\tau})\right\|^{2} d \hat{\tau} \leq\left\|\phi_{y}\left(\tau_{0}\right)\right\|^{2}+\int_{\tau_{0}}^{\tau}\left\|\psi_{y}(\hat{\tau})\right\|^{2} d \hat{\tau} \\
& +\int_{\tau_{0}}^{\tau} \int_{ \pm}\left(\phi_{\tau} \phi_{y}\right)_{y} d \hat{\tau} \quad+C\left\{\int _ { \tau _ { 0 } } ^ { \tau } \int _ { \pm } \left(\left|\phi_{y y}\right|\left(\left|\varphi_{y} \phi_{y}\right|+\left|\psi_{y}^{2}\right|\right)\right.\right. \\
& +\left|U_{y} \phi_{y y}\right|(|\phi|+|\varphi|)+\left|\phi_{y y}\right|\left(\left|V_{y} \phi_{y}\right|+\left|\Theta_{y} \varphi_{y}\right|\right. \\
& \left.\left.\left.\quad+\left|U_{y} \psi_{x}\right|\right)+\left|\phi_{y y}\right|\left(\left|U_{y y}\right|+\left|U_{y}^{2}\right|\right)\right)(y, \hat{\tau}) d y d \hat{\tau}\right\}
\end{aligned}
$$

where the right hand side can be estimated as follows,

$$
\begin{gathered}
\int_{\tau_{0}}^{\tau} \int_{ \pm}\left(\phi_{\tau} \phi_{y}\right)_{y} d y d \hat{\tau}=\left.\left[\phi \phi_{y}\right]\right|_{\tau_{0}} ^{\tau}-\int_{\tau_{0}}^{\tau} \phi\left[\phi_{y}\right]_{\tau}=\left.\phi\left[\phi_{y}\right]\right|_{\tau_{0}} ^{\tau}-\int_{\tau_{0}}^{\tau} \phi\left[\phi_{y}\right]_{\tau} \\
\leq \frac{1}{16} \int_{\tau_{0}}^{\tau}\left\|\phi_{y y}(\hat{\tau})\right\|_{ \pm}^{2} d \hat{\tau}+C \int_{\tau_{0}}^{\tau}\left(\|\psi\|^{2}+\|\phi\|^{2}+\left\|\phi_{y}\right\|_{ \pm}^{2}\right)(\hat{\tau}) d \hat{\tau} ; \\
\int_{\tau_{0}}^{\tau} \int_{ \pm}\left|\left(\varphi_{y} \phi_{y} \phi_{y y}\right)(y, \hat{\tau})\right| d y d \hat{\tau} \leq C \int_{\tau_{0}}^{\tau}\left\|\varphi_{y}(\hat{\tau})\right\|_{ \pm}\left\|\phi_{y}(\hat{\tau})\right\|_{ \pm}^{1 / 2}\left\|\phi_{y y}(\hat{\tau})\right\|_{ \pm}^{3 / 2} d \hat{\tau} \\
\leq \frac{1}{16} \int_{\tau_{0}}^{\tau}\left\|\phi_{y y}(\hat{\tau})\right\|_{ \pm}^{2} d \hat{\tau}+C N\left(\tau_{0}, \tau\right) \int_{\tau_{0}}^{\tau}\left\|\phi_{y}(\hat{\tau})\right\|_{ \pm}^{2} d \hat{\tau} ; \\
\int_{\tau_{0}}^{\tau} \int_{ \pm}\left|\left(\psi_{y}^{2} \phi_{y y}\right)(y, \hat{\tau})\right| d y d \hat{\tau} \leq C \int_{\tau_{0}}^{\tau}\left\|\psi_{y}(\hat{\tau})\right\|_{ \pm}^{3 / 2}\left\|\psi_{y y}(\hat{\tau})\right\|_{ \pm}^{1 / 2}\left\|\phi_{y y}(\hat{\tau})\right\|_{ \pm} d \hat{\tau} \\
\leq \frac{1}{16} \int_{\tau_{0}}^{\tau}\left\|\phi_{y y}(\hat{\tau})\right\|_{ \pm}^{2} d \hat{\tau}+C N\left(\tau_{0}, \tau\right) \int_{\tau_{0}}^{\tau}\left(\left\|\psi_{y y}(\hat{\tau})\right\|_{ \pm}^{2}+\left\|\psi_{y}(\hat{\tau})\right\|_{ \pm}^{2}\right) d \hat{\tau} ; \\
\int_{\tau_{0}}^{\tau} \int_{ \pm}\left|U_{y} \phi_{y y}\right|(|\varphi|+|\phi|)(y, \hat{\tau}) d y d \hat{\tau} \\
\left.\leq \frac{1}{16} \int_{\tau_{0}}^{\tau}\left\|\phi_{y y}(\hat{\tau})\right\|_{ \pm}^{2} d \hat{\tau}+C \delta \int_{\tau_{0}}^{\tau}\left\|\sqrt{V_{\tau}}(\varphi, \phi)(\hat{\tau})\right\|\right) d \hat{\tau} ; \\
\int_{\tau_{0}}^{\tau} \int_{ \pm}\left|\phi_{y y}\right|\left(\left|V_{y} \phi_{y}\right|+\left|\Theta_{y} \varphi_{y}\right|+\left|U_{y} \psi_{y}\right|\right)(y, \hat{\tau}) d y d \hat{\tau} \\
\left.\leq \frac{1}{16} \int_{\tau_{0}}^{\tau}\left\|\phi_{y y}(\hat{\tau})\right\|_{ \pm}^{2} d \hat{\tau}+C \delta \int_{\tau_{0}}^{\tau}\left\|\left(\varphi_{y}, \psi_{y}, \phi_{y}\right)(\hat{\tau})\right\|_{ \pm}\right) d \hat{\tau}
\end{gathered}
$$


and

$$
\begin{aligned}
& \left.\int_{\tau_{0}}^{\tau} \int_{ \pm} \mid \phi_{y y}\right) \mid\left(\left|U_{y y}\right|+\left|U_{y}^{2}\right|\right)(y, \hat{\tau}) d y d \hat{\tau} \leq \frac{1}{16} \int_{\tau_{0}}^{\tau}\left\|\phi_{y y}(\hat{\tau})\right\|_{ \pm}^{2} d \hat{\tau} \\
& \quad+C \int_{\tau_{0}}^{\tau}\left(\left\|U_{y y}\right\|^{2}+\left\|U_{y}\right\|_{L^{4}}^{4}\right)(\hat{\tau}) d \hat{\tau} \leq \frac{1}{16} \int_{\tau_{0}}^{\tau}\left\|\phi_{y y}(\hat{\tau})\right\|_{ \pm}^{2} d \hat{\tau}+C \delta^{1 / 4}
\end{aligned}
$$

Substitution of the above estimates into (3.13) gives Lemma 3.4 immediately.

Now, combining Lemmas 3.1-3.4, we obtain Proposition 2.6.

Acknowledgments. The authors would like to thank the referees for their suggestions which improve the presentation of the present paper.

\section{REFERENCES}

[1] S. Bianchini And A. Bressan, Vanishing viscosity solutions of nonlinear hyperbolic systems, Ann. of Math., 161 (2005), pp. 223-342.

[2] A. Bressan, BV-Solutions to Hyperbolic Systems By Vanishing Viscosity, S.I.S.S.A., Trieste, Italy, 2000.

[3] A. BREssan AND T. YANG, On the convergence rate of vanishing viscosity approximations, Comm. Pure Appl. Math., 57 (2004), pp. 1075-1109.

[4] R. Courant and K.O. Friedrichs, Supersonic Flows and Shock Waves, Wiley-Interscience, New York, 1948.

[5] C.M. Dafermos, Hyperbolic Conservation Laws in Continuum Physics, Springer-Verlag, Berlin, Heidelberg, 2000.

[6] X.Q. Ding, G.-Q. Chen, And P. Luo, Convergence of the Lax-Friedrich scheme for the system of equations of isentropic gas dynamics. I, Acta Mathematica Scientia, 7 (1987), pp. 467480 (in Chinese).

[7] R.J. DiPerna, Convergence of approximate solutions to conservation laws, Arch. Rat. Mech. Anal., 83 (1983), pp. 27-70.

[8] R.J. DiPerna, Convergence of the viscosity method for isentropic gas dynamics, Comm. Math. Phys., 91 (1983), pp. 1-30.

[9] J. Goodman And Z. XIn, Viscous limits for piecewise smooth solutions to systems of conservation laws, Arch. Rat. Mech. Anal., 121 (1992), pp. 235-265.

[10] E. Grenier and F. Rousset, Stability of one-dimensional boundary layers by using Green's functions, Comm. Pure Appl. Math., 54 (2001), pp. 1343-1385.

[11] O. Gùes, G. MÉtivier, M. Williams, and K. Zumbrun, Multidimensional viscous shocks II: The small viscosity limit, Comm. Pure Appl. Math., 57 (2004), pp. 141-218.

[12] O. Gùes, G. Métivier, M. Williams, and K. Zumbrun, A new approach to the stability of multidimensional viscous shocks, Preprint 2004.

[13] D. HofF, Global well-posedness of Cauchy problem for Navier-Stokes equations of nonisentropic flow with discontinuous initial data, J. Diff. Eqs., 95 (1992), pp. 33-74.

[14] D. Hoff AND T.P. LiU, The inviscid limit for Navier-Stokes equations of compressible isentropic flow with shock data, Indiana Univ. Math. J., 38 (1989), pp. 861-915.

[15] L. HSIAO AND R.H. PAN, Zero relaxation limit to centered rarefaction waves for a rate-type viscoelastic system, J. Diff. Eqs., 157 (1999), pp. 20-40.

[16] S. Kawashima, A. Matsumura, and K. Nishinara, Asymptotic behaviour of of solutions for the equations of a viscous heat-conductive gas, Proc. Japan Acad. Ser. A, 62 (1986), pp. $249-252$.

[17] P.L. Lions, B. Perthame, and P.E. Souganidis, Existence and stability of entropy solutions for the hyperbolic systems of isentropic gas dynamics in Eulerian and Lagrangian coordinates, Comm. Pure Appl. Math., 49 (1996), 599-638.

[18] P.L. Lions, B. Perthame, And E. Tadmor, Kinetic formulation of the isentropic gas dynamics and p-system, Comm. Math. Phys., 163 (1994), pp. 415-431.

[19] T.P. LiU AND Z. XIN, Nonlinear stability of rarefaction waves for compressible Navier-Stokes equations, Comm. Math. Phys., 118 (1988), pp. 451-465.

[20] A. Matsumura And K. Nishinara, Asymptotics toward the rarefaction waves of the solutions of a one-dimensional model system for compressible viscous gas, Japan J. Appl. Math., 3 (1986), pp. 1-13. 
[21] A. Matsumura and K. Nishinara, Global stability of the rarefaction waves of a onedimensional model system for compressible viscous gas, Comm. Math. Phys., 144 (1992), pp. 325-335.

[22] K. Nishinara, T. YANG, AND H.J. ZhaO, Nonlinear stability of strong rarefaction waves for compressible Navier-Stokes equations, SIAM J. Math. Anal., 35 (2004), pp. 1561-1597.

[23] F. Rousset, Stability of small amplitude boundary layers for mixed hyperbolic-parabolic systems, Trans. Amer. Math. Soc., 355 (2003), pp. 2991-3008.

[24] D. Serre, Systems of Conservation Laws I,II, Cambridge Univ. Press, 2000.

[25] D. Serre, Sur la stabilité des couches limites de viscosité, Ann. Inst. Fourier, 51 (2001), pp. $109-129$.

[26] D. Serre and K. Zumbrun, Boundary layer stability in real vanishing viscosity limit, Comm. Math. Phys., 221 (2001), pp. 267-292.

[27] J. Smoller, Shock Waves and Reactive Diffusion Equations, Springer-Verlag, New York, Berlin, Heidelberg, 1983.

[28] W.C. WANG AND Z. XIN, Fluid-dynamic limit for the centered rarefaction wave of the Broadwall equation, J.Diff. Eqs., 150 (1998), pp. 438-461.

[29] Y. WANG AND Z. XIN, Zero-viscosity limit of the linearized compressible Navier-Stokes equations with highly oscillatory forces in the half-plane, IMS Preprint No. 2004-08, CUHK, Hong Kong.

[30] Z. XIN, Zero dissipation limit to rarefaction waves for the one-dimensional Navier-Stokes equations of compressible isentropic gases, Comm. Pure Appl. Math., 46 (1993), pp. 621665.

[31] Z. XIN, Viscous boundary layers and their stability. I, J. Partial Diff. Eqs., 11 (1998), pp. 97-124.

[32] Z. Xin And T. Yanagisawa, Zero-viscosity limit of the linearized Navier-Stokes equations for a compressible viscous fluid in the half-plane, Comm. Pure Appl. Math., 52 (1999), pp. 479-541.

[33] S. YU, Zero-dissipation limit of solutions with shocks for systems of hyperbolic conservation laws, Arch. Rat. Mech. Anal., 146 (1999), pp. 275-370. 